\section{Ginkgo biloba did not prevent dementia or Alzheimer disease in elderly people}

\section{QUESTION}

Does Ginkgo biloba reduce incident dementia and Alzheimer disease in elderly people with normal cognition or mild cognitive impairment?

\section{METHODS}

Design: randomised placebo controlled trial (Ginkgo Evaluation of Memory [GEM] study). ClinicalTrials.gov NCT00010803

Allocation: unclear allocation concealment. *

Blinding: blinded (patients, clinicians, and outcome assessors).*

Follow-up period: median 6.1 years.

Setting: 5 academic medical centres in the USA.

Participants: 3069 participants $>75$ years of age (mean age $79 \mathrm{y}, 54 \%$ men) who had normal cognition or mild cognitive impairment (impaired at $\leqslant 10^{\text {th }}$ percentile of Cardiovascular Health Study normative data on 2 of 10 neuropsychological tests and Clinical Dementia Rating global score of 0.5). Exclusion criteria included dementia; bleeding disorders; Parkinson disease; receipt of warfarin, cholinesterase inhibitors, antidepressants, or antipsychotics; abnormal thyroid tests, serum creatinine concentration $>2 \mathrm{mg} / \mathrm{dl}$ $(>176.8 \mu \mathrm{mol} / \mathrm{l})$, or liver function test result $>2$ times the upper limit of normal; or allergy to Ginkgo biloba.
Intervention: Ginkgo biloba, $120 \mathrm{mg}$ twice daily ( $\mathrm{n}=1545)$, or matching placebo $(\mathrm{n}=1524)$.

Outcomes: diagnosis of dementia and Alzheimer disease.

Patient follow-up: 94\% (intention-to-treat analysis).

\section{MAIN RESULTS}

Ginkgo biloba and placebo did not differ for incident dementia or Alzheimer disease (table).

\section{CONCLUSION}

Ginkgo biloba did not prevent incident dementia or Alzheimer disease in elderly people with normal cognition or mild cognitive impairment.

\section{ABSTRACTED FROM}

Dekosky ST, Williamson JD, Fitzpatrick AL, et al for the Ginkgo Evaluation of Memory (GEM) Study Investigators. Ginkgo biloba for prevention of dementia: a randomized controlled trial. JAMA 2008;300:2253-62.

Correspondence to: Dr S T DeKosky, University of Virginia School of Medicine, Charlottesville,VA, USA; dekosky@virginia.edu

Sources of funding: National Center for Complementary and Alternative Medicine; Office of Dietary Supplements; National Institute on Aging; National Heart, Lung, and Blood Institute; University of Pittsburgh Alzheimer's Disease Research Center: Roena Kulynych Center for Memory and Cognition Research; National Institute of Neurological Disorders and Stroke; Schwabe Pharmaceuticals donated Ginkgo biloba tablets and placebos.

Clinical impact ratings: Elderly care 6/7; Family/general practice 6/7; Psychiatry $6 / 7$

Ginkgo biloba $v$ placebo for preventing dementia in elderly people*

\begin{tabular}{lllll}
\hline & & & \multicolumn{2}{c}{ At a median $\mathbf{6 . 1}$ years } \\
\cline { 4 - 5 } Outcomes & Ginkgo biloba & Placebo & RRI (95\% CI) & NNH \\
\hline Dementia & $18 \%$ & $16 \%$ & $11 \%(-6$ to 29$)$ & Not significant \\
Alzheimer disease & $17 \%$ & $14 \%$ & $15 \%(-3$ to 35$)$ & Not significant \\
\hline
\end{tabular}

${ }^{*}$ Abbreviations defined in glossary. $\mathrm{RRI}, \mathrm{NNH}$, and $\mathrm{Cl}$ calculated from control event rates and hazard ratios in article. inkgo biloba is a popular herbal supplement that is widely used in Europe and the USA for both treating and preventing dementia. However, good quality evidence supporting its effectiveness is lacking. In 2007, a Cochrane review of 35 clinical trials ( $n=4247$ ) suggested that Ginkgo biloba was ineffective for dementia. ${ }^{1}$ The study by DeKosky et al shows that Ginkgo biloba is no more effective than placebo for preventing dementia. It addresses many weaknesses identified in previous studies by virtue of its large size $(n=3069)$ and relatively lengthy duration ( $\geqslant 6$ y exposure to Ginkgo biloba or placebo).

People often consider herbal therapies to be safe, even if they are not effective, and although I have previously suggested that financial harm occurs if people spend money on ineffectual remedies, ${ }^{2}$ this study suggests that physical harm may also occur. There was a non-significant increase in haemorrhagic strokes in the intervention group, and although this may be due to chance alone, it raises the question of "how safe is Ginkgo biloba?" This is an important question as the perception exists that herbal remedies are intrinsically safer than pharmaceutical preparations. ${ }^{3}$ The clinical bottom line is that no evidence exists to support use of this supplement to prevent dementia. This fits into the broader picture that Ginkgo biloba has no effect on slowing down progression of dementia in the early stages of disease.
Gareth Parsons, RGN, MSc, PGCE(FE), RNT HeSAS, University of Glamorgan

Pontypridd, Wales, UK

1. Birks J, Grimley Evans J. Ginkgo biloba for cognitive impairment and dementia. Cochrane Database Syst Rev 2007:(2):CD003120.

2. Parsons G. Commentary on "Review: evidence does not support use of static magnets for pain." Evid Based Nurs 2008:11:49. Comment on: Pittle $\mathrm{MH}$, Brown EM, Ernst E. Static magnets for reducing pain: systematic review and metaanalysis of randomized trials. CMAJ 2007;177 736-42.

3. Elvin-Lewis $\mathbf{M}$. Should we be concerned about herbal remedies. J Ethnopharmacol 2001;75: 141-64 